Review

\title{
Notch and breast cancer metastasis
}

Yu Zhang1, Huan Deng 2, 3, 4,*

1 The first clinical medical college of Nanchang University, Nanchang 330000, China;

2 Department of Pathology, The Fourth Affiliated Hospital of Nanchang University, Nanchang 330000, China;

3 Molecular Medicine and Genetics Center, The Fourth Affiliated Hospital of Nanchang University, Nanchang 330000, China;

${ }^{4}$ Renmin Institute of Forensic Medicine in Jiangxi, Nanchang 330000, China

* Correspondence: e-mail@beandeng@ncu.edu.cn; Tel.: +86 79187022537

\begin{abstract}
Metastasis is a major cause factor for breast cancer (BC)-associated mortality. During the metastatic process, disseminated tumor cells (DTCs) detach from the primary sites, and enter the bloodstream and establish the secondary colonies. Recent studies have provided substantial evidence for the importance of Notch signaling in BC metastasis. Therefore, this review focuses on the mechanisms by which Notch contributes to the origin of BC DTCs, increases their motility, regulates their intravasation and extravasation, protects them from host surveillance, and finally facilitates colonization. Identification of the mechanisms underlying Notch-related BC metastasis will lead to the development of novel Notch-targeted therapeutic strategies to reduce metastasis and significantly improve outcomes.
\end{abstract}

Key words: breast cancer; metastasis; Notch; Jagged-1; Dll4

\section{Background}

The incidence of breast cancer (BC), by far the most common cancer in females, has steadily increased in much of the world over the last few decades [1, 2]. BC-associated mortality is typically due to distant metastasis, rather than due to the primary tumor. Despite recent advances in the application of targeted therapeutic strategies, significant improvements in the prognosis of patients with metastatic $\mathrm{BC}$ have not yet been observed due to the poor understanding of the molecular mechanisms underlying the metastatic process.

Metastasis is a complicated cascade involving interactions between cancer cells and surrounding microenvironmental components such as mesenchymal cells, immune cells, and the extracellular matrix. The first stage of $\mathrm{BC}$ metastasis is characterized by the invasion of the basement membrane by primary tumor cells, which then become disseminated tumor cells (DTCs). These cells then promote abnormal angiogenesis, intravasate into the circulatory or lymphatic system, head towards distant organs, and establish secondary tumors.

Accumulating evidence indicates the important role of Notch, a highly conserved family of signaling molecules, in BC metastasis. The deregulation of Notch signaling is reflected in all aspects of the metastatic processes and its role in $\mathrm{BC}$ seems to be highly context dependent.

\section{Notch signaling}

The mammalian Notch family consists of four receptors (Notch 1-4), each of which includes an extracellular domain (ECD), a transmembrane domain and an intracellular domain (ICD). Notch ligands in Drosophila have two homologs: Delta and Serrate. Their counterparts in mammals are Delta-like molecules (DLLs) and Jagged [3].

Activation of Notch signaling includes receptor and ligand binding, cleavage of the ICD, and translocation of activated Notch ICD (NICD) into the nucleus. The receptor undergoes two cleavages following ligand-ECD binding. First, the S2 site is cleaved by tumor necrosis factor- $\alpha$ converting enzyme (TACE, also called ADAM17) to release the ECD[4]. Second, $\gamma$-secretase 
cleaves the S3 site to release NICD, which requires the involvement of Presenilin [5]. Then, NICD

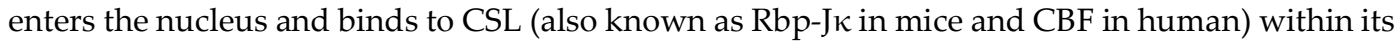
RAM region[6].

\section{Notch and BC tumorigenesis}

\subsection{Biased cell fate}

Symmetric divisions (contribute to normal replication and amplification) and asymmetric divisions (contribute to mammary gland morphogenesis, bi-potent mammary stem cells (MaSCs) divide into luminal or basal stem cells, and then become ductal/alveolar or myoepithelial cells respectively) help to maintain the balance between proliferation, self-renewal, and differentiation in breast cells[7].

Notch signaling contributes to MaSCs biological behaviors[8]. CBF disruption in mammary gland during pregnancy can lead to a luminal to basal fate switch and stronger expansion of basal cell population[9]. While the developmental decisions of Notch signaling is controlled by Numb, a protein asymmetrically segregated in dividing progenitor cells. Numb facilitates Notch ubiquitination at the membrane, promotes degradation of NICD, circumvents its nuclear translocation and inhibits Notch signaling downstream activation[10].

However, involvement of Notch signaling in the disorder of BC stem cells is also of great importance due to its overexpression. once balance is disturbed, MaSCs steady state numbers may be disrupted, allowing mutant stem cells to arise, compete with existing stem cells, and establish tumors. E74-Like Factor 5 (ELF5) regulates differentiation into the mammary alveolar and luminal lineages. It also inhibits BC metastasis by suppressing the activation of Slug (a transcription factor that drives the epithelial-mesenchymal transition (EMT)) and loss of which provides a fertile ground for tumorigenesis[11]. Notch1 gene contains ELF-5 responsive elements. NICD1 and NICD4, are hyperactivated in ELF5-null mammary epithelial cells[12]. Thus, hyperactivation of Notch pathway may be a potent driver for the ELF-5-null BC phenotype.

\section{Notch and local invasion}

\subsection{Invasion of the basement membrane and mesenchyme}

Specific gene programs equip cancer cells with increasrd mobility, driving their migration away from primary sites.

EMT is central to the regulation of epithelial plasticity and cancer cell mobility. During this cascade process, epithelial cells lose their cellular polarity and adhesion junctions but acquire mesenchymal characteristics. In vitro studies have suggested that Notch1 knockdown can reverse the Jagged1-induced EMT. These Notch1-silenced cells are capable of a less aggressive form of invasion characterized by a cobblestone-shaped phenotype rather than a spindle-like mesenchymal phenotype [13]. Notably, many studies have demonstrated that Jagged1-mediated Notch activation suppresses the expression of E-cadherin and increase levels of mesenchymal markers Vimentin and N-cadherin, and the transcription factors Slug, Snail and Zeb1, as well as $\beta$-catenin in BC cells, can translocate to the nucleus and promote migration and invasion [14-19].

Notch1 can activate EMT by inducing STAT3 and upregulating the expression of p65 and IL1 [20]. Further, Notch2 has been found to promote EMT through the IL-6/JAK/STAT3 pathway in a radiation-driven model of BC EMT[21]. In the more aggressive triple-negative breast cancer (TNBC), loss of Numb can activate Notch signaling and induce both EMT and CSC-like properties, leading to early relapse and metastasis[22, 23]. Other mobility-promoting programs, including F-actin polymerization can also be induced by Notch1[24].

Enhanced migratory ability by itself, however, is not sufficient to drive metastasis. Disseminating cancer cells must also invade the surrounding complex network, which mainly consists of ECM, basement membrane, and mesenchyme. The matrix metalloproteinase (MMP) 
family is known to degrade the ECM and promote cancer cell invasion and metastasis[25]. In BC, Notch1 activation mostly promotes MMP-2 and MMP-9 expression to break down the elements of ECM[26]. Urokinase-type plasminogen activator (uPA) is an ECM-degradating enzyme associated with poor outcomes and a high risk of metastasis and recurrence[27]. Under normal conditions, uPA induces a plasminogen proteolytic sequence. However, in BC, uPA works with MMPs to erode the microvasculature and degrade the ECM to facilitate the tumor cell metastasis. uPA receptor (uPAR) is highly expressed in malignant tissues and tends to be located at the leading edge or invasion front. Upon binding to uPA, the receptor converts plasminogen to plasmin[28]. Recent studies place Notch upstream of the uPA cascade[29]. A positive link has been observed between Jagged-1 and uPA in various BC cell lines, and Notch 1knockdown reduces uPA levels. Furthermore, Notch may directly regulate uPA transcription via CBF-1 binding sites within the uPA promoter and enhancer. The subsequently activated uPAR can then cleave ECM-related signaling molecules including fibronectin and the laminin receptor[29, 30].

The low oxygen environment (hypoxia) resulting from leaky vasculature and a lack of blood supply is important for tumor progression. Hypoxia-inducible factor $1 \alpha$ (HIF-1 $\alpha$ ) can promote metastasis and is associated with poor prognosis [31-33]. Accumulation of HIF-1 $\alpha$ and HIF-2 $\alpha$ enhance Notch signaling (both ligands and receptors) as well as the expression of following genes HES1 and HEY1. HIFs and MAML1 (a key Notch co-activator) form a complex with NICD to recruit other Notch co-activators such as p300, indicating a hypoxia/(HIF/MAML1)/Notch axis. Hypoxia stabilizes HIFs through this signaling cascade, resulting in elevated Notch[14].

Hypoxia-induced Notch activation causes EMT in BC. First, NICD can directly bind to Snail1 promoter. Second, Notch can potentiate HIF-1 $\alpha$ to bind to the lysyl oxidase (LOX) promoter and then stabilizes the secretion of Snail- 1 and tissue inhibitor of metalloproteinase 4 (TIMP4), leading to EMT and metastasis [34, 35]. Consistent with its ability to simulate ECM degradation, LOX mostly correlates with ER (estrogen receptor)-negative breast cancer, which is more likely to metastasize to bone rather than ER-positive $\mathrm{BC}[36]$. The ability of LOX to induce metastasis indicates that it may be a potential target to prevent bone metastasis of breast cancer.

\subsection{The interaction between $B C$ cells and stromal cells}

The tumor microenvironment mainly consisting of mesenchymal and immune cells, is central to $\mathrm{BC}$ progression. Solid experimental evidence indicates that cancer-associated fibroblasts (CAFs) can secrete cytokines to support $\mathrm{BC}$ cells and protect them from host surveillance. CAFs can secrete ADAM10-rich exosomes (recently found to be associated with loss of Timp family member expression[37]) to potentiate cell motility and aldehyde dehydrogenase expression through RhoA and Notch, respectively[37]. In turn, silencing of Notch effector Rbp-J $\kappa$, combining with down-regulation of p53, induces a senescent phenotype and the expression of CAF-effector genes [38].

4.3. Angiogenesis

The angiogenic phenotype is necessary for malignant growth. Vascular endothelial growth factor (VEGF) is the most important mitogenic factor that stimulates the formation of vascular endothelial cells and recruits perivascular support cells to establish a neovasculature; this allows for the entry of DTCs and transport of nutrition factors[39]. Endothelial Notch signaling is also essential for tumor angiogenesis.

Notch ligands exert opposite effects on angiogenesis. The ends of vessel sprouts are made of tip cells, while the other cells are called stalk cells; both cell types are essential for vessel polarity and barrier function of vessels[40]. This endothelial cell specification is regulated by Notch signaling. Upregulation of DLL4 by VEGF in endothelial tip cells suppresses the tip-like phenotype. Conversely, DLL4 knock-out leads to excessive tip cell formation. Vessel sprouting is also dramatically augmented by DLL4 inhibition. However, this process by itself cannot lead to tumor angiogenesis because the dysfunctional, immature vasculature have a reduced lumen size and are thus mostly non-productive and can actually repress tumor growth[41]. Accumulating 
evidence attributes tumor inhibition to poor perfusion and Angiopoietin1 (Ang1)-induced vascular quiescence[42].

Another ligand, Jagged-1 mainly exists in stalk cells. Overexpression of Jagged-1 results in a hybrid tip/stalk phenotype, thus transforming angiogenesis from a physiological to pathological pattern[43]. Jagged-1 regulates the function of angiocrine through Notch3/Hey1 to promote EMT as well as DTC proliferation and survival[44]. Interestingly, Jagged-1 can attenuate DLL4mediated inhibition of VEGFR2 and VEGFR3 through its pro-angiogenic function, indicating a "tug-of-war" that maintains a balance between VEGFR isoform[45].

Demethylases and methyltransferases dynamically regulate the histone methylation in $\mathrm{BC}$. Expression of the lysine demethylase 2A (KDM2A) is increased in human BC and is correlated with poor prognosis. Jagged-1 is a direct downstream target of KDM2A and is essential for KDM2A-driven tumor angiogenesis. Inhibition of KDM2A in BC cells blocks Notch activation and endothelial cell tube formation [46].

Notch-related proteases also participate in angiogenesis. MMPs can make space for angiogenesis and lymphangiogenesis[25]. Additionally, uPA and UPAR together can active VEGF [29].

\subsection{Intravasation}

Notch signaling modulates the ability of BC cells to cross both mesenchymal and endothelial barriers. Different integrins have been associated with both normal mammary epithelial cells and BC cells. Integrin $\beta 1$ inhibits the expression of Notch [47]. Meanwhile, activation of Notch signaling can also control $\beta 1$ integrin affinity, suggesting a feedback loop between Notch and integrins [48]. $\beta 1$ integrin cooperates with Notch to promote the transendothelial migration of BC cells, which is characterized by enhanced polarity reversal and adhesion to the blood vessel wall $[49,50]$.

Aberrant Notch activation stimulates endothelial cells (ECs) to promote BC intravasation. Vascular cell adhesion molecule-1 (VCAM1) can be subverted by Notch1 to enhance the adhesion of tumor cells and neutrophils to ECs, thus favoring the dissemination of tumor cells[51]. As mentioned above, BC cells can also recruit factors such as MMPs that increase vascular permeability and thus promote intravasation Figure.a. 


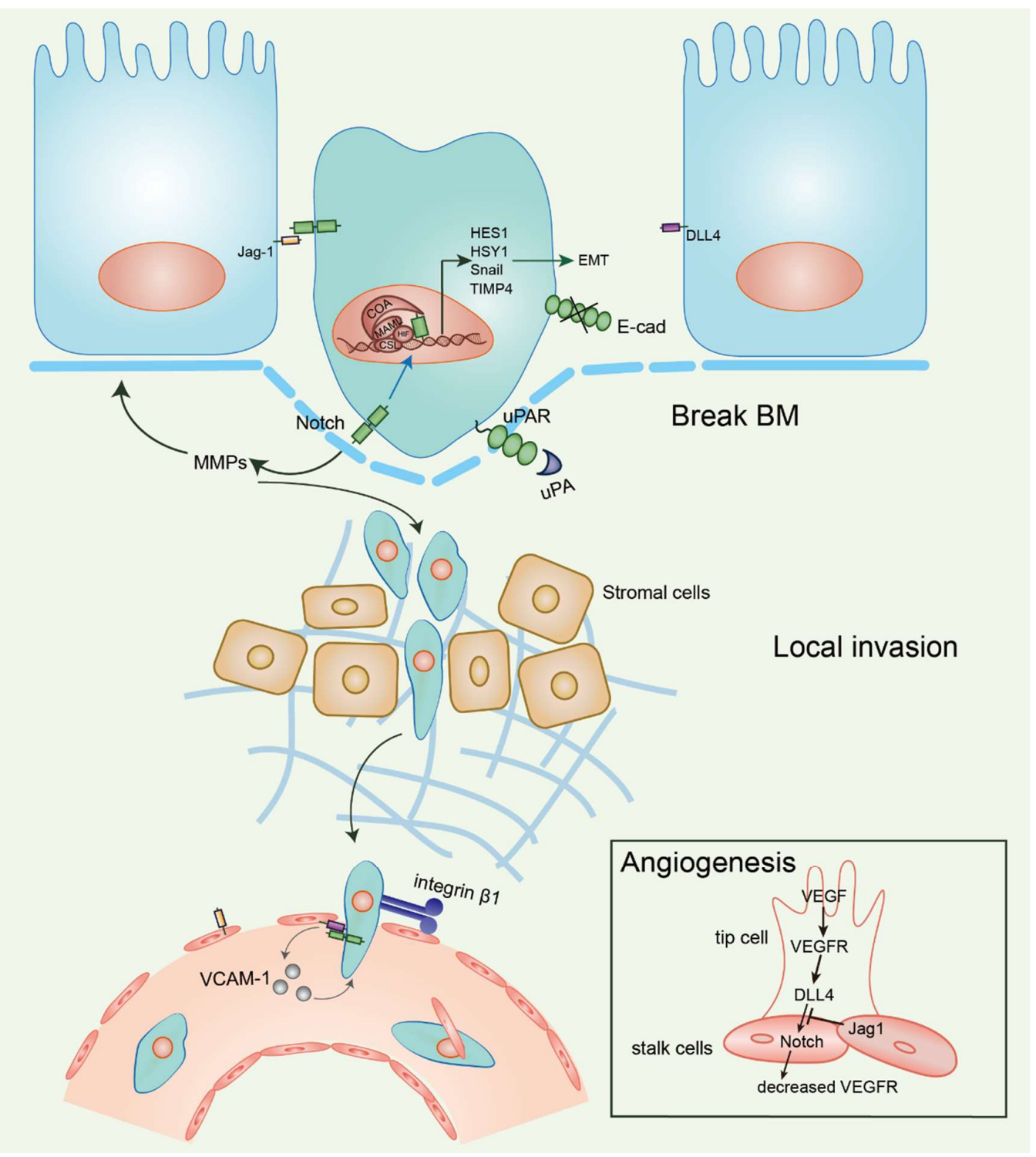

Figure. a BC DTCs detach from primary sites and intravasation. They obtain increased motility to and break basement membrane and overcome ECM with the Notch-related processes such as hypoxia and EMT as well as enzymes including MMPs and uPA. Notch takes part in angiogenesis to sprout new vessels and BC DTCs can enter bloodstream through these vasculatures 


\section{Notch and survival in circulation}

BC cells detach from primary sites and then enter the circulation, becoming circulating tumor cells (CTCs). DTCs must first survive in the bloodstream before they can arrive at distant organs. 5.1. Anti-apoptosis

Apoptosis negatively regulates tumor progression by preventing overgrowth. This process depends on the coordination of many ligands and receptors including tumor necrosis factorrelated apoptosis-inducing ligand (TRAIL)/TRAIL-receptor1 and 2 (also called DR4 and DR5). Administration of GSIs ( $\gamma$-secretase inhibitors) can strongly upregulate DR4 and DR5, increase the sensitization of BC cells to TRAIL-mediated apoptosis[52], active the caspase system (caspase8 here)[53], promoting mitochondrial membrane leakiness and further inducing apoptosis. This Notch-mediated anti-apoptosis function may depend on AP1, a dimeric transcription factor complex constituted by c-Jun. Blockage by GSIs, failed cleavage of Notch increases the level of AP1 and JNK (the enzyme responsible for c-Jun activation) [52, 54, 55], thus actives DR4 and DR5[56]. A recent study showed that Notch4, but not Notch1, is involved in the sensitization of TRAIL [57]. Alternatively, inhibition of $\beta 1$ integrins can sensitize tumor cells to TRAIL-induced apoptosis, which is mediated by Notch[58]. The GSI/TRAIL combination also decrease several survival factors like survivin and Bcl-2. Moreover, diverse types of BC cells differ in their response to such inhibition. For example, ER-negative $B C$ cells are more sensitive to GSI/TRAIL synergism than ER-positive cells [52].

AKT impedes DNA damage induced-apoptosis through the inhibition of apoptosis signalregulating kinase 1 (ASK1) and prevents JNK-mediated activation of p53[59], leading to an aberrant increase in the number of mammary stem and progenitor cells [60]. Substantial evidence has demonstrated that impairment of Notch signaling can both inhibit AKT activity and sensitize cells to apoptosis[59, 61]. Notch can also directly bind the amino terminus of p53 independent of the presence of AKT, thus inhibiting p53 phosphorylation and DNA binding activity[62].

Notch-mediated regulation of AKT contributes to tumor cell survival through multiple pathways. AKT can increase MMP production via several downstream target proteins including NF- $\kappa B$ and mammalian target of rapamycin (mTOR)[63]. Li et al [26] suggests that Notch1 inhibition can enhance protein phosphatase $2 \mathrm{~A}(\mathrm{PP} 2 \mathrm{~A})$ activity and downregulate NF- $\mathrm{kB}$, which can be restored by PP2A inhibitor, okadaic acid (OA). Treatment with OA also upregulates VEGF, MMP2, and MMP9, suggesting a key role of PP2A in the Notch/AKT/NF- $k B$ axis. mTOR also takes part in AKT-mediated tumor cell survival, which contributes to chemoresistance. Its downstream effector, eukaryotic initiation factor $4 \mathrm{E}$ (eIF4E), is crucial for mTOR-mediated inhibition of p53 and can reverse p53-mediated cytotoxicity[64].

\subsection{Chemoresistance}

DTCs must employ several elegant approaches to overcome the threat of chemotherapy. To contend with Adriamycin or paclitaxel, two first-line chemotherapy agents for BC, DTCs may acquire morphological and functional endothelial features characteristic of tumor vascularization. DLL3 and Notch4, together with their downstream targets p65 (an NF- $\kappa$ B subunit) and Zeb1, are overexpressed in tumor-derived endothelial cells during chemotherapy. Silencing of Notch4/DLL3 can decrease the functionality of tumor-derived endothelial cells and endothelial markers. The expression of VEGFR3 (important in tumor angiogenesis) is significantly upregulated in patients receiving chemotherapy. Notch4/DLL3 silencing can also suppress the expression of VEGFR3 transcripts, indicating that BC chemotherapy triggers the formation of functional tumor-derived endothelial microvessels by regulating Notch signaling and VEGF signaling [65].

ER-positive BC patients with high levels of aldehyde dehydrogenase 1 (ALDH1) and Notch4 exhibit poor prognosis following anti-estrogen treatment. Although short-term treatment does 
suppress tumor cell proliferation, it also increases DTC activity through Jagged-1-Notch4 activation. Administration of Notch inhibitors tends to attenuate drug resistance and improve overcomes [66]. Long-term hormonal therapies can reduce ER $\alpha$ expression and increase the IL-6 levels, thus enhancing the self-renewal properties of hormonal therapy-resistant tumor cells (both ER-dependent and ER-independent). Further studies have demonstrated that hormonal therapy initially abolishes oxidative phosphorylation (OXPHOS) to generate self-renewal-deficient cells. Then, IL-6 cause a departure from metabolic dormancy induced by mitochondrial activation through IL-6/STAT3/Notch3 transduction pattern, hence gaining resistance[67]. STAT1 can also facilitate the expansion of therapy-resistant BC cells through Notch3 [68].

Interestingly, multiple courses of treatment for ER-positive/human epidermal growth factor receptor

2 (HER2)-negative BC patients can endow their CTCs with HER2 expression[69]. Further study demonstrated that BC CTCs which exhibit this transformation maintain discrete HER2-positive and HER2-negative subpopulations. More importantly, HER2-positive and HER2-negative BC CTCs interconvert spontaneously but function differently. HER2-positive CTCs acquire a stronger proliferation potential and a higher lung metastasis frequency but no more sensitive to HER2-targered therapy. While HER2-negative CTCs show an increased level of Notch1 but a resistance to chemotherapy. Therefore, dual treatment in chemotherapy-sensitive HER2positive/Notch1-negative and Notch inhibitor-sensitive HER2-negative/Notch-positive CTCs makes sense[70].

\section{Notch and secondary colonization}

Prior to establishing metastases in secondary organs, BC CTCs release factors including MMPs and gather bone marrow-derived hematopoietic precursor cells (HPCs) to combine with perivascular fibroblasts and fibronectin (FN), to form the pre-metastatic niche. Once DTCs (or CTCs) extravasate and colonize the niche, they become micrometastases[71].

The mechanism underlying metastatic organotropism is still unknown; specifically, studies have been unable to provide a rationale for the propensity of $\mathrm{BC}$ to metastasize to the bone, lung and liver. But several evidences reveal bone metastasis of BC with help of Notch. In bone, BC cells employ many signaling pathways, including Notch, to mediate osteoblast activation and differentiation [72]. Bone marrow osteoblasts produce TGF- $\beta$, which increases levels of the Notch signaling proteins Notch3 and Jagged1, thus promoting the secretion of osteoblast-derived IL-6 and osteoblast differentiation. Inhibition of Notch signaling via knockdown of Notch3 or treatment with a $\gamma$-secretase inhibitor markedly reduces BC bone metastasis[73, 74], Figure. b.

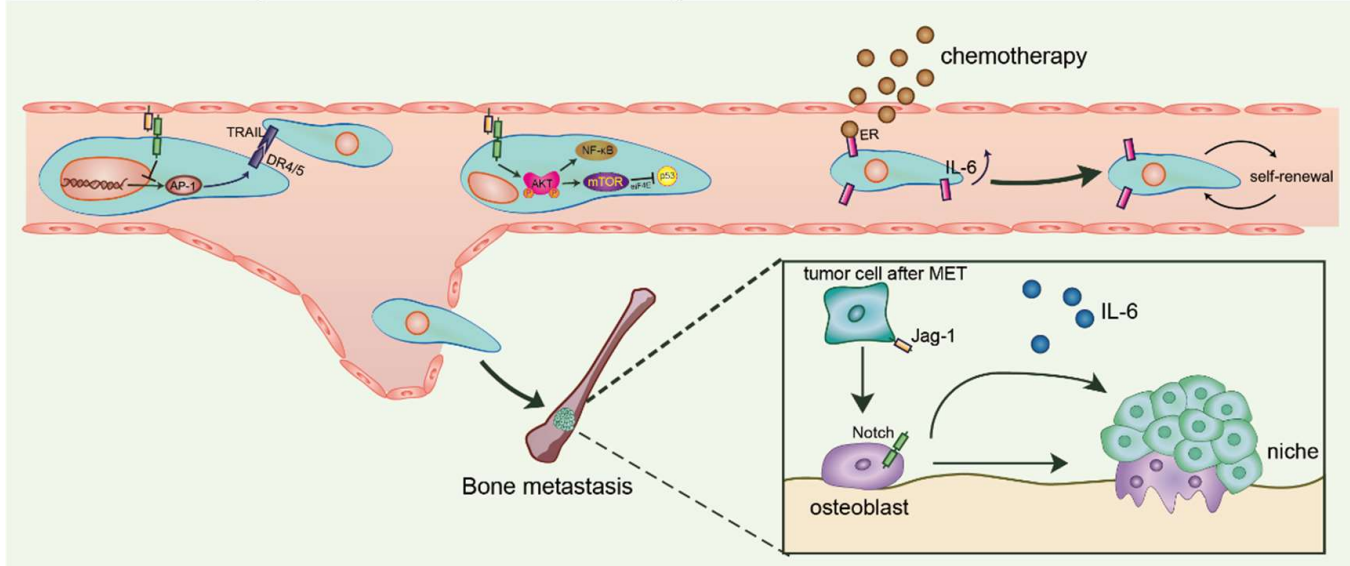

Figure. b BC CTCs survives in bloodstream and colonize in bone. Notch helps BC CTCs to survive from TRAIL apoptosis systems and tumor suppressor gene p53. Chemotherapies increases IL-6 level by IL-6/STAT3/Notch3 axis to enhance self-renewal property of ER+BCs. Notch also 
facilitates bone metastasis of BCs by regulating osteoblasts activation and differentiation

KiSS1, a metastasis suppressor gene, is downregulated in BC secondary tumor sites[75]. By enhancing $\mathrm{I}-\kappa \mathrm{B}$ activation, KiSS1 prevents NF- $\kappa \mathrm{B}$ binding to pro-inflammatory and prometastatic gene promoters, thus potentially competing with Notch. KiSS1 encodes a COOHterminally amidated active peptide, metastin, whose ligand is a novel G protein-coupled receptor. Interestingly, metastin only affects secondary tumor sites rather than primary lesions [76].

$\mathrm{Nm} 23$ is also a suppressor of BC metastasis[77]. The Nm23 protein, particularly the Nm23H1 isoform, has three main targets: ATP citrate lyase (involved in metabolism); aldolase C (involved in hypoxia); and kinase suppressor of Ras (KSR) (involved in regulation of mitogenic activity). Meanwhile, Nm23 can suppress several metastasis-related factors such as Smoothened (a key receptor in Hedgehog signaling) and pleiotrophin [71]. Treatment with nonsteroidal antiinflammatory drugs (NSAIDs), such as aspirin, can upregulate Nm23 expression, thus inhibiting Notch/HES1 and reducing CTCs [78, 79]. Ignesti et al suggested that loss of Drosophila awd, a homolog of Nm23, might inhibit Notch signaling after S2 cleavage [80].

Expression of Tenascin C (TNC), an ECM protein that is located in the stem cell niche, is a potential biomarker for breast CTCs that have infiltrated the lung[81]. TNC enhances the expression of musashi homolog 1 (MSI1), a regulator of Notch signaling, and thus confers breast CTCs with enhanced migratory and invasive properties [82]. Moreover, TNC can also prevent MSI1-mediated Notch signaling through the inhibition of STAT5. Thus, high levels of MSI1, Notch1, and Jagged1 are associated with poor prognosis of patients with BC [83, 84]. More importantly, cancer-induced sprouting neovasculature can promote tip cells to secret POSTN (POSTN associates with Notch1 physically at its EGF repeats $[85,86]$ ) to bind to TNC and ECM and help TNC deposition on the ECM and incorporation into the ECM [87].

It is incumbent on DTCs to expand and establish new colonies. Otherwise, these cells will enter dormancy, which is defined as growth arrest or a balance between proliferation and apoptosis [88]. Dormant breast DTCs in the lung may be experiencing an absence of uPA- and $\alpha 5 \beta 1$ integrin-triggered proliferative signaling[89]. Reactivation of dormancy calls for increased uPAR- $\alpha 5 \beta 1$ integrin complexes and activation of upstream Notch signaling. Notch3 is responsible for the stability of MKP-1 (mitogen-activated protein kinase phosphatase-1), a widely-expressed phosphatase. Previous reports have demonstrated that levels of Notch3 and MKP-1 are quite low in dormant tumors, resulting in high levels of phosphorylated p38, a target of MKP-1 that contributes to the maintaince of dormancy[90].

\section{Conclusion and clinical development}

It is clear that Notch signaling has many roles in tumor metastasis. Exploring of only one pathway may be insufficient for the development of appropriate therapeutic since the activation of related pathways as well as the cross-talk between Notch and other signaling pathways (such as wingless-type MMTV integration site (WNT)) is of critical importance. Specific aspects that will be improtant to consider include TNC stimulation of both Notch and WNT signaling to balance dormancy and quiescence make sense [82].

Current treatments for metastatic BC are mainly palliative with little clinical effectiveness[91]. Encouragingly, many studies over the last few years have focused on the treatment of metastatic BC via targeting Notch signaling[92]. $\gamma$-secretase inhibitors show great potential. PF-03084014 (Pfizer Oncology), a small molecule selective noncompetitive and reversible GSI, displays synergistic activity with docetaxel and has demonstrated significant antitumor activity in triple-negative BC patients[93]. Moreover, a potent non-competitive oral GSI, MK-0752 (MERK), has been evaluated for the treatment of metastatic BC via induction of G0/G1 arrest[94]. Monoclonal antibodies against DLL4, such as OMP-21M18 (OncoMed Pharmaceuticals) and REGN421/SAR153192 (Regeneron Pharmaceuticals) as well as OMP-59R5 
and OPM-52M51, which target Notch $2 / 3$ and Notch 1 receptors, respectively, are also in clinical trials[92].

Though many drugs are in development, enormous challenges remain before a Notchtargeted therapeutic strategy can be clinically applied. BC patients receiving continuous doses of MK-0752 at $450 \mathrm{mg}$ daily present significant symptoms of toxicity and fatigue[95]. Further, gastrointestinal toxicity is also a major side effect in patients being treated with GSIs[96].

Despite surgical resection of primary tumors and subsequent chemotherapy and radiotherapy, recurrence at distant organs suggests that dissemination of tumor cells may occur at very early, typically asymptomatic, stages. Advanced detection technology allows for very early diagnosis of metastasis, even when very few tumor cells have disseminated to the peripheral blood or bone marrow. Interestingly, some patients carrying DTCs never develop metastasis while other patients with large metastases do not present with DTCs at the time of primary tumor detection. Thus, the complicated mechanisms of metastasis reveal just the tip of the iceberg, and our knowledge of Notch signaling and BC metastasis is nowhere near enough.

\section{Compliance with Ethical Standards}

Funding: This study was funded by the grant from the National Science Foundation of China (81770624), Foundation of Jiangxi Educational Committee (No. GJJ160048), and Foundation of Health and Family Planning Commission of Jiangxi Province (No. 20171098 and 20161086).

Conflicts of Interest: Yu Zhang declares that he has no conflict of interest. Huan Deng declares that he has no conflict of interest.

Ethical approval: This article does not contain any studies with human participants or animals performed by any of the authors.

\section{Reference}

1. $\quad$ Siegel, R. L., K. D. Miller, and A. Jemal. "Cancer Statistics, 2017." CA Cancer J Clin 67, no. 1 (2017): 730 .

2. Chen, W., R. Zheng, P. D. Baade, S. Zhang, H. Zeng, F. Bray, A. Jemal, X. Q. Yu, and J. He. "Cancer Statistics in China, 2015." CA Cancer J Clin 66, no. 2 (2016): 115-32.

3. Bray, S. J. "Notch Signalling: A Simple Pathway Becomes Complex." Nat Rev Mol Cell Biol 7, no. 9 (2006): 678-89.

4. Brou, C., F. Logeat, N. Gupta, C. Bessia, O. LeBail, J. R. Doedens, A. Cumano, P. Roux, R. A. Black, and A. Israel. "A Novel Proteolytic Cleavage Involved in Notch Signaling: The Role of the DisintegrinMetalloprotease Tace." Mol Cell 5, no. 2 (2000): 207-16.

5. Sprinzak, D., A. Lakhanpal, L. Lebon, L. A. Santat, M. E. Fontes, G. A. Anderson, J. Garcia-Ojalvo, and M. B. Elowitz. "Cis-Interactions between Notch and Delta Generate Mutually Exclusive Signalling States." Nature 465, no. 7294 (2010): 86-90.

6. Wu, L., J. C. Aster, S. C. Blacklow, R. Lake, S. Artavanis-Tsakonas, and J. D. Griffin. "Maml1, a Human Homologue of Drosophila Mastermind, Is a Transcriptional Co-Activator for Notch Receptors." Nat Genet 26, no. 4 (2000): 484-9.

7. Santoro, A., T. Vlachou, M. Carminati, P. G. Pelicci, and M. Mapelli. "Molecular Mechanisms of Asymmetric Divisions in Mammary Stem Cells." EMBO Rep 17, no. 12 (2016): 1700-20.

8. Bouras, T., B. Pal, F. Vaillant, G. Harburg, M. L. Asselin-Labat, S. R. Oakes, G. J. Lindeman, and J. E. Visvader. "Notch Signaling Regulates Mammary Stem Cell Function and Luminal Cell-Fate Commitment." Cell Stem Cell 3, no. 4 (2008): 429-41. 
9. Buono, K. D., G. W. Robinson, C Martin, S. Shi, P Stanley, K Tanigaki, T Honjo, and L Hennighausen. "The Canonical Notch/Rbp-J Signaling Pathway Controls the Balance of Cell Lineages in Mammary Epithelium During Pregnancy." Developmental Biology 293, no. 2 (2006): 565-80.

10. McGill, M. A., and C. J. McGlade. "Mammalian Numb Proteins Promote Notch1 Receptor Ubiquitination and Degradation of the Notch1 Intracellular Domain." J Biol Chem 278, no. 25 (2003): 23196-203.

11. Chakrabarti, Rumela, Julie Hwang, Mario Andres Blanco, Yong Wei, Martin Lukačišin, Rose Anne Romano, Kirsten Smalley, Song Liu, Qifeng Yang, and Toni Ibrahim. "Elf5 Inhibits Epithelial Mesenchymal Transition in Mammary Gland Development and Breast Cancer Metastasis by Transcriptionally Repressing Snail2/Slug." Nat Cell Biol 14, no. 11 (2012): 1212-22.

12. Chakrabarti, R., Y. Wei, R. A. Romano, C. DeCoste, Y. Kang, and S. Sinha. "Elf5 Regulates Mammary Gland Stem/Progenitor Cell Fate by Influencing Notch Signaling." Stem Cells 30, no. 7 (2012): 1496-508.

13. Shao, S., X. Zhao, X. Zhang, M. Luo, X. Zuo, S. Huang, Y. Wang, S. Gu, and X. Zhao. "Notch1 Signaling Regulates the Epithelial-Mesenchymal Transition and Invasion of Breast Cancer in a Slug-Dependent Manner." Mol Cancer 14 (2015): 28.

14. Chen, J., N. Imanaka, J. Chen, and J. D. Griffin. "Hypoxia Potentiates Notch Signaling in Breast Cancer Leading to Decreased E-Cadherin Expression and Increased Cell Migration and Invasion." Br J Cancer 102, no. 2 (2010): 351-60.

15. Leong, K. G., K. Niessen, I. Kulic, A. Raouf, C. Eaves, I. Pollet, and A. Karsan. "Jagged1-Mediated Notch Activation Induces Epithelial-to-Mesenchymal Transition through Slug-Induced Repression of ECadherin." J Exp Med 204, no. 12 (2007): 2935-48.

16. Jian, J., Q. Yang, Y. Shao, D. Axelrod, J. Smith, B. Singh, S. Krauter, L. Chiriboga, Z. Yang, J. Li, and X. Huang. "A Link between Premenopausal Iron Deficiency and Breast Cancer Malignancy." BMC Cancer 13 (2013): 307.

17. Liu, L., X. Chen, Y. Wang, Z. Qu, Q. Lu, J. Zhao, X. Yan, H. Zhang, and Y. Zhou. "Notch3 Is Important for Tgf-Beta-Induced Epithelial-Mesenchymal Transition in Non-Small Cell Lung Cancer Bone Metastasis by Regulating Zeb-1." Cancer Gene Ther 21, no. 9 (2014): 364-72.

18. Bolos, V., E. Mira, B. Martinez-Poveda, G. Luxan, M. Canamero, A. C. Martinez, S. Manes, and J. L. de la Pompa. "Notch Activation Stimulates Migration of Breast Cancer Cells and Promotes Tumor Growth." Breast Cancer Res 15, no. 4 (2013): R54.

19. Brabletz, S., K. Bajdak, S. Meidhof, U. Burk, G. Niedermann, E. Firat, U. Wellner, A. Dimmler, G. Faller, J. Schubert, and T. Brabletz. "The Zeb1/Mir-200 Feedback Loop Controls Notch Signalling in Cancer Cells." Embo j 30, no. 4 (2011): 770-82.

20. Zhang, X., X. Zhao, S. Shao, X. Zuo, Q. Ning, M. Luo, S. Gu, and X. Zhao. "Notch1 Induces EpithelialMesenchymal Transition and the Cancer Stem Cell Phenotype in Breast Cancer Cells and Stat3 Plays a Key Role." Int J Oncol 46, no. 3 (2015): 1141-8.

21. Kim, R. K., N. Kaushik, Y. Suh, K. C. Yoo, Y. H. Cui, M. J. Kim, H. J. Lee, I. G. Kim, and S. J. Lee. "Radiation Driven Epithelial-Mesenchymal Transition Is Mediated by Notch Signaling in Breast Cancer." Oncotarget 7, no. 33 (2016): 53430-42.

22. Zhang, J., X. Shao, H. Sun, K. Liu, Z. Ding, J. Chen, L. Fang, W. Su, Y. Hong, H. Li, and H. Li. "Numb Negatively Regulates the Epithelial-Mesenchymal Transition of Triple-Negative Breast Cancer by Antagonizing Notch Signaling." Oncotarget 7, no. 38 (2016): 61036-53.

23. Garcia-Heredia, J. M., E. M. Verdugo Sivianes, A. Lucena-Cacace, S. Molina-Pinelo, and A. Carnero. "Numb-Like (Numbl) Downregulation Increases Tumorigenicity, Cancer Stem Cell-Like Properties and Resistance to Chemotherapy." Oncotarget 7, no. 39 (2016): 63611-28.

24. Wang, J., L. Fu, F. Gu, and Y. Ma. "Notch1 Is Involved in Migration and Invasion of Human Breast Cancer Cells." Oncol Rep 26, no. 5 (2011): 1295-303.

25. Kessenbrock, K., V. Plaks, and Z. Werb. "Matrix Metalloproteinases: Regulators of the Tumor Microenvironment." Cell 141, no. 1 (2010): 52-67.

26. Li, L., F. Zhao, J. Lu, T. Li, H. Yang, C. Wu, and Y. Liu. "Notch-1 Signaling Promotes the Malignant Features of Human Breast Cancer through Nf-Kappab Activation." PLoS One 9, no. 4 (2014): e95912. 
27. Mahmood, Niaz, Catalin Mihalcioiu, and Shafaat A Rabbani. "Multifaceted Role of the Urokinase-Type Plasminogen Activator (Upa) and Its Receptor (Upar): Diagnostic, Prognostic, and Therapeutic Applications." Front Oncol 8 (2018): 24.

28. Heiss, M. M., H. Allgayer, K. U. Gruetzner, I. Funke, R. Babic, K. W. Jauch, and F. W. Schildberg. "Individual Development and Upa-Receptor Expression of Disseminated Tumour Cells in Bone Marrow: A Reference to Early Systemic Disease in Solid Cancer." Nat Med 1, no. 10 (1995): 1035-9.

29. Shimizu, M., B. Cohen, P. Goldvasser, H. Berman, C. Virtanen, and M. Reedijk. "Plasminogen Activator Upa Is a Direct Transcriptional Target of the Jag1-Notch Receptor Signaling Pathway in Breast Cancer." Cancer Res 71, no. 1 (2011): 277-86.

30. Song, Jifeng. "Notch Signaling Mediates Tumor-Caf Crosstalk in Basal-Like Breast Cancer." Master (2014).

31. Gupta, G. P., and J Massagué. "Cancer Metastasis: Building a Framework." Cell 127, no. 4 (2006): 67995.

32. Semenza, Gregg L. "Hif-1 and Tumor Progression: Pathophysiology and Therapeutics." Trends in Molecular Medicine 8, no. 4 Suppl (2002): S62-7.

33. Harris, A. L. "Hypoxia--a Key Regulatory Factor in Tumour Growth." Nat Rev Cancer 2, no. 1 (2002): 38-47.

34. Boufraqech, M., L. Zhang, N. Nilubol, S. M. Sadowski, S. Kotian, M. Quezado, and E. Kebebew. "Lysyl Oxidase (Lox) Transcriptionally Regulates Snai2 Expression and Timp4 Secretion in Human Cancers." Clin Cancer Res 22, no. 17 (2016): 4491-504.

35. Sahlgren, C., M. V. Gustafsson, S. Jin, L. Poellinger, and U. Lendahl. "Notch Signaling Mediates Hypoxia-Induced Tumor Cell Migration and Invasion." Proc Natl Acad Sci U S A 105, no. 17 (2008): 6392-7.

36. Villanueva, M. T. "Metastasis: Lox Does Some Prepping." Nat Rev Cancer 15, no. 7 (2015): 384.

37. Shimoda, M., S. Principe, H. W. Jackson, V. Luga, H. Fang, S. D. Molyneux, Y. W. Shao, A. Aiken, P. D. Waterhouse, C. Karamboulas, F. M. Hess, T. Ohtsuka, Y. Okada, L. Ailles, A. Ludwig, J. L. Wrana, T. Kislinger, and R. Khokha. "Loss of the Timp Gene Family Is Sufficient for the Acquisition of the CafLike Cell State." Nat Cell Biol 16, no. 9 (2014): 889-901.

38. Procopio, M. G., C. Laszlo, D. Al Labban, D. E. Kim, P. Bordignon, S. H. Jo, S. Goruppi, E. Menietti, P. Ostano, U. Ala, P. Provero, W. Hoetzenecker, V. Neel, W. W. Kilarski, M. A. Swartz, C. Brisken, K. Lefort, and G. P. Dotto. "Combined Csl and P53 Downregulation Promotes Cancer-Associated Fibroblast Activation." Nat Cell Biol 17, no. 9 (2015): 1193-204.

39. Hanahan, D., and J. Folkman. "Patterns and Emerging Mechanisms of the Angiogenic Switch During Tumorigenesis." Cell 86, no. 3 (1996): 353-64.

40. Hellstrom, M., L. K. Phng, J. J. Hofmann, E. Wallgard, L. Coultas, P. Lindblom, J. Alva, A. K. Nilsson, L. Karlsson, N. Gaiano, K. Yoon, J. Rossant, M. L. Iruela-Arispe, M. Kalen, H. Gerhardt, and C. Betsholtz. "Dll4 Signalling through Notch1 Regulates Formation of Tip Cells During Angiogenesis." Nature 445, no. 7129 (2007): 776-80.

41. Jia, Xuelian, Wenyi Wang, Zhuobin Xu, Shijing Wang, Tong Wang, Min Wang, and Min Wu. "A Humanized Anti-Dll4 Antibody Promotes Dysfunctional Angiogenesis and Inhibits Breast Tumor Growth." Sci Rep 6 (2016): 27985.

42. Zhang, J., S. Fukuhara, K. Sako, T. Takenouchi, H. Kitani, T. Kume, G. Y. Koh, and N. Mochizuki. "Angiopoietin-1/Tie2 Signal Augments Basal Notch Signal Controlling Vascular Quiescence by Inducing Delta-Like 4 Expression through Akt-Mediated Activation of Beta-Catenin." J Biol Chem 286, no. 10 (2011): 8055-66.

43. Boareto, M., M. K. Jolly, E. Ben-Jacob, and J. N. Onuchic. "Jagged Mediates Differences in Normal and Tumor Angiogenesis by Affecting Tip-Stalk Fate Decision." Proc Natl Acad Sci U S A 112, no. 29 (2015): E3836-44.

44. Pedrosa, A. R., A. Trindade, C. Carvalho, J. Graca, S. Carvalho, M. C. Peleteiro, R. H. Adams, and A. Duarte. "Endothelial Jagged1 Promotes Solid Tumor Growth through Both Pro-Angiogenic and Angiocrine Functions." Oncotarget 6, no. 27 (2015): 24404-23. 
45. Benedito, R., C. Roca, I. Sorensen, S. Adams, A. Gossler, M. Fruttiger, and R. H. Adams. "The Notch Ligands Dll4 and Jagged1 Have Opposing Effects on Angiogenesis." Cell 137, no. 6 (2009): 1124-35.

46. Chen, J. Y., C. F. Li, P. Y. Chu, Y. S. Lai, C. H. Chen, S. S. Jiang, M. F. Hou, and W. C. Hung. "Lysine Demethylase 2a Promotes Stemness and Angiogenesis of Breast Cancer by Upregulating Jagged1." Oncotarget 7, no. 19 (2016): 27689-710.

47. Deford, P., K. Brown, R. L. Richards, A. King, K. Newburn, K. Westover, and A. R. Albig. "Magp2 Controls Notch Via Interactions with Rgd Binding Integrins: Identification of a Novel Ecm-IntegrinNotch Signaling Axis." Exp Cell Res 341, no. 1 (2016): 84-91.

48. Hodkinson, P. S., P. A. Elliott, Y Lad, B. J. Mchugh, A. C. Mackinnon, C Haslett, and T Sethi. "Mammalian Notch-1 Activates Beta1 Integrins Via the Small Gtpase R-Ras." Journal of Biological Chemistry 282, no. 39 (2007): 28991-9001.

49. Liu, Bingbing, Xia Zheng, Fanfan Meng, Yunwei Han, Yawen Song, Fangfang Liu, Shuai Li, Lanjing Zhang, Feng Gu, and Xinmin Zhang. "Overexpression of B1 Integrin Contributes to Polarity Reversal and a Poor Prognosis of Breast Invasive Micropapillary Carcinoma." Oncotarget 9, no. 4 (2018): 433853.

50. Stoletov, Konstantin, Hisashi Kato, Erin Zardouzian, Jonathan Kelber, Jing Yang, Sanford Shattil, and Richard Klemke. "Visualizing Extravasation Dynamics of Metastatic Tumor Cells." Journal of Cell Science 123, no. 13 (2010): 2332-41.

51. Guo, P., and S. Rafii. "Dangerous Liaisons: Deviant Endothelium Notches toward Tumor Metastasis." Cancer Cell 31, no. 3 (2017): 301-03.

52. Portanova, P., A. Notaro, O. Pellerito, S. Sabella, M. Giuliano, and G. Calvaruso. "Notch Inhibition Restores Trail-Mediated Apoptosis Via Ap1-Dependent Upregulation of Dr4 and Dr5 Trail Receptors in Mda-Mb-231 Breast Cancer Cells." Int J Oncol 43, no. 1 (2013): 121-30.

53. Day, Travis W., Su Huang, and Ahmad R. Safa. "C-Flip Knockdown Induces Ligand-Independent Dr5, Fadd-, Caspase-8-, and Caspase-9-Dependent Apoptosis in Breast Cancer Cells." Biochem Pharmacol 76, no. 12 (2008): 1694-704.

54. Kim, J. W., M. J. Kim, K. J. Kim, H. J. Yun, J. S. Chae, S. G. Hwang, T. S. Chang, H. S. Park, K. W. Lee, and P. L. Han. "Notch Interferes with the Scaffold Function of Jnk-Interacting Protein 1 to Inhibit the Jnk Signaling Pathway." Proc Natl Acad Sci U S A 102, no. 40 (2005): 14308.

55. Archibald, A., C. Mihai, I. G. Macara, and L. McCaffrey. "Oncogenic Suppression of Apoptosis Uncovers a Rac1/Jnk Proliferation Pathway Activated by Loss of Par3." Oncogene 34, no. 24 (2015): 3199-206.

56. Zou, W., X. Liu, P. Yue, Z. Zhou, M. B. Sporn, R Lotan, F. R. Khuri, and S. Y. Sun. "C-Jun Nh2-Terminal Kinase-Mediated up-Regulation of Death Receptor 5 Contributes to Induction of Apoptosis by the Novel Synthetic Triterpenoid Methyl-2-Cyano-3,12-Dioxooleana-1, 9-Dien-28-Oate in Human Lung Cancer Cells." Cancer Research 64, no. 20 (2004): 7570-78.

57. Naik, S., M. MacFarlane, and A. Sarin. "Notch4 Signaling Confers Susceptibility to Trail-Induced Apoptosis in Breast Cancer Cells." J Cell Biochem 116, no. 7 (2015): 1371-80.

58. Phipps, L. E., S Hino, and R. J. Muschel. "Targeting Cell Spreading: A Method of Sensitizing Metastatic Tumor Cells to Trail-Induced Apoptosis." Molecular Cancer Research 9, no. 3 (2011): 249-58.

59. Meurette, O., S. Stylianou, R. Rock, G. M. Collu, A. P. Gilmore, and K. Brennan. "Notch Activation Induces Akt Signaling Via an Autocrine Loop to Prevent Apoptosis in Breast Epithelial Cells." Cancer Res 69, no. 12 (2009): 5015-22.

60. Tao, L., A. L. Roberts, K. A. Dunphy, C. Bigelow, H. Yan, and D. J. Jerry. "Repression of Mammary Stem/Progenitor Cells by P53 Is Mediated by Notch and Separable from Apoptotic Activity." Stem Cells 29, no. 1 (2011): 119-27.

61. Su, F., S. Zhu, J. Ruan, Y. Muftuoglu, L. Zhang, and Q. Yuan. "Combination Therapy of Ry10-4 with the Gamma-Secretase Inhibitor Dapt Shows Promise in Treating Her2-Amplified Breast Cancer." Oncotarget 7, no. 4 (2016): 4142-54. 
62. Kim, S. B., G. W. Chae, J. Lee, J. Park, H. Tak, J. H. Chung, T. G. Park, J. K. Ahn, and C. O. Joe. "Activated Notch1 Interacts with P53 to Inhibit Its Phosphorylation and Transactivation." Cell Death Differ 14, no. 5 (2007): 982-91.

63. Hay, N. "The Akt-Mtor Tango and Its Relevance to Cancer." Cancer Cell 8, no. 3 (2005): 179-83.

64. Mungamuri, S. K., X. Yang, A. D. Thor, and K. Somasundaram. "Survival Signaling by Notch1: Mammalian Target of Rapamycin (Mtor)-Dependent Inhibition of P53." Cancer Res 66, no. 9 (2006): 4715-24.

65. Zhang, P., D. He, Z. Chen, Q. Pan, F. Du, X. Zang, Y. Wang, C. Tang, H. Li, H. Lu, X. Yao, J. Jin, and X. Ma. "Chemotherapy Enhances Tumor Vascularization Via Notch Signaling-Mediated Formation of Tumor-Derived Endothelium in Breast Cancer." Biochem Pharmacol 118 (2016): 18-30.

66. Simoes, B. M., C. S. O'Brien, R. Eyre, A. Silva, L. Yu, A. Sarmiento-Castro, D. G. Alferez, K. Spence, A. Santiago-Gomez, F. Chemi, A. Acar, A. Gandhi, A. Howell, K. Brennan, L. Ryden, S. Catalano, S. Ando, J. Gee, A. Ucar, A. H. Sims, E. Marangoni, G. Farnie, G. Landberg, S. J. Howell, and R. B. Clarke. "AntiEstrogen Resistance in Human Breast Tumors Is Driven by Jag1-Notch4-Dependent Cancer Stem Cell Activity." Cell Rep 12, no. 12 (2015): 1968-77.

67. Sansone, P., C. Ceccarelli, M. Berishaj, Q. Chang, V. K. Rajasekhar, F. Perna, R. L. Bowman, M. Vidone, L. Daly, J. Nnoli, D. Santini, M. Taffurelli, N. N. Shih, M. Feldman, J. J. Mao, C. Colameco, J. Chen, A. DeMichele, N. Fabbri, J. H. Healey, M. Cricca, G. Gasparre, D. Lyden, M. Bonafe, and J. Bromberg. "Self-Renewal of Cd133(Hi) Cells by Il6/Notch3 Signalling Regulates Endocrine Resistance in Metastatic Breast Cancer." Nat Commun 7 (2016): 10442.

68. Boelens, M. C., T. J. Wu, B. Y. Nabet, B. Xu, Y. Qiu, T. Yoon, D. J. Azzam, C. Twyman-Saint Victor, B. Z. Wiemann, H. Ishwaran, P. J. Ter Brugge, J. Jonkers, J. Slingerland, and A. J. Minn. "Exosome Transfer from Stromal to Breast Cancer Cells Regulates Therapy Resistance Pathways." Cell 159, no. 3 (2014): 499-513.

69. Arteaga, C. L., and J. A. Engelman. "Erbb Receptors: From Oncogene Discovery to Basic Science to Mechanism-Based Cancer Therapeutics." Cancer Cell 25, no. 3 (2014): 282-303.

70. Jordan, N. V., A. Bardia, B. S. Wittner, C. Benes, M. Ligorio, Y. Zheng, M. Yu, T. K. Sundaresan, J. A. Licausi, R. Desai, R. M. O'Keefe, R. Y. Ebright, M. Boukhali, S. Sil, M. L. Onozato, A. J. Iafrate, R. Kapur, D. Sgroi, D. T. Ting, M. Toner, S. Ramaswamy, W. Haas, S. Maheswaran, and D. A. Haber. "Her2 Expression Identifies Dynamic Functional States within Circulating Breast Cancer Cells." Nature 537, no. 7618 (2016): 102-06.

71. Mack, G. S., and A. Marshall. "Lost in Migration." Nat Biotechnol 28, no. 3 (2010): 214-29.

72. Kang, Y. "Dissecting Tumor-Stromal Interactions in Breast Cancer Bone Metastasis." Endocrinol Metab (Seoul) 31, no. 2 (2016): 206-12.

73. Zhang, Z., H. Wang, S. Ikeda, F. Fahey, D. Bielenberg, P. Smits, and P. V. Hauschka. "Notch3 in Human Breast Cancer Cell Lines Regulates Osteoblast-Cancer Cell Interactions and Osteolytic Bone Metastasis." Am J Pathol 177, no. 3 (2010): 1459-69.

74. Sethi, N., X. Dai, C. G. Winter, and Y. Kang. "Tumor-Derived Jagged1 Promotes Osteolytic Bone Metastasis of Breast Cancer by Engaging Notch Signaling in Bone Cells." Cancer Cell 19, no. 2 (2011): 192-205.

75. Lee, J. H., and D. R. Welch. "Suppression of Metastasis in Human Breast Carcinoma Mda-Mb-435 Cells after Transfection with the Metastasis Suppressor Gene, Kiss-1." Cancer Res 57, no. 12 (1997): 2384-7.

76. Ohtaki, T., Y. Shintani, S. Honda, H. Matsumoto, A. Hori, K. Kanehashi, Y. Terao, S. Kumano, Y. Takatsu, Y. Masuda, Y. Ishibashi, T. Watanabe, M. Asada, T. Yamada, M. Suenaga, C. Kitada, S. Usuki, T. Kurokawa, H. Onda, O. Nishimura, and M. Fujino. "Metastasis Suppressor Gene Kiss-1 Encodes Peptide Ligand of a G-Protein-Coupled Receptor." Nature 411, no. 6837 (2001): 613-7.

77. Leone, A, U Flatow, K Vanhoutte, and P. S. Steeg. "Transfection of Human Nm23-H1 into the Human Mda-Mb-435 Breast Carcinoma Cell Line: Effects on Tumor Metastatic Potential, Colonization and Enzymatic Activity." Oncogene 8, no. 9 (1993): 2325. 
78. Yu, H. G., J. A. Huang, Y. N. Yang, H. Huang, H. S. Luo, J. P. Yu, J. J. Meier, H. Schrader, A. Bastian, W. E. Schmidt, and F. Schmitz. "The Effects of Acetylsalicylic Acid on Proliferation, Apoptosis, and Invasion of Cyclooxygenase-2 Negative Colon Cancer Cells." Eur J Clin Invest 32, no. 11 (2002): 838-46.

79. Moon, C. M., J. H. Kwon, J. S. Kim, S. H. Oh, K. Jin Lee, J. J. Park, S. Pil Hong, J. H. Cheon, T. I. Kim, and W. H. Kim. "Nonsteroidal Anti-Inflammatory Drugs Suppress Cancer Stem Cells Via Inhibiting Ptgs2 (Cyclooxygenase 2) and Notch/Hes1 and Activating Pparg in Colorectal Cancer." Int J Cancer 134, no. 3 (2014): 519-29.

80. Ignesti, M., M. Barraco, G. Nallamothu, J. A. Woolworth, S. Duchi, G. Gargiulo, V. Cavaliere, and T. Hsu. "Notch Signaling During Development Requires the Function of Awd, the Drosophila Homolog of Human Metastasis Suppressor Gene Nm23." BMC Biol 12 (2014): 12.

81. Oskarsson, Thordur, Swarnali Acharyya, Xiang Hf Zhang, Sakari Vanharanta, Sohail F Tavazoie, Patrick G Morris, Robert J Downey, Katia Manovatodorova, Edi Brogi, and Joan Massagué. "Breast Cancer Cells Produce Tenascin C as a Metastatic Niche Component to Colonize the Lungs." Nature Medicine 17, no. 7 (2011): 867-74.

82. Oskarsson, T., S. Acharyya, X. H. Zhang, S. Vanharanta, S. F. Tavazoie, P. G. Morris, R. J. Downey, K. Manova-Todorova, E. Brogi, and J. Massague. "Breast Cancer Cells Produce Tenascin C as a Metastatic Niche Component to Colonize the Lungs." Nat Med 17, no. 7 (2011): 867-74.

83. Reedijk, M., S. Odorcic, L. Chang, H. Zhang, N. Miller, D. R. McCready, G. Lockwood, and S. E. Egan. "High-Level Coexpression of Jag1 and Notch1 Is Observed in Human Breast Cancer and Is Associated with Poor Overall Survival." Cancer Res 65, no. 18 (2005): 8530-7.

84. Wang, X. Y., L. O. Penalva, H. Yuan, R. I. Linnoila, J. Lu, H. Okano, and R. I. Glazer. "Musashi1 Regulates Breast Tumor Cell Proliferation and Is a Prognostic Indicator of Poor Survival." Mol Cancer 9 (2010): 221.

85. Tanabe, H., I. Takayama, T. Nishiyama, M. Shimazaki, I. Kii, M. Li, N. Amizuka, K. Katsube, and A. Kudo. "Periostin Associates with Notch1 Precursor to Maintain Notch1 Expression under a Stress Condition in Mouse Cells." PLoS One 5, no. 8 (2010): e12234.

86. Zhou, M., N. Kawashima, N. Suzuk, M. Yamamoto, K. Ohnishi, K. Katsube, H. Tanabe, A. Kudo, M. Saito, and H. Suda. "Periostin Is a Negative Regulator of Mineralization in the Dental Pulp Tissue." Odontology 103, no. 2 (2015): 152-9.

87. Kii, I., T. Nishiyama, M. Li, K. Matsumoto, M. Saito, N. Amizuka, and A. Kudo. "Incorporation of Tenascin-C into the Extracellular Matrix by Periostin Underlies an Extracellular Meshwork Architecture." J Biol Chem 285, no. 3 (2010): 2028-39.

88. Aguirre-Ghiso, J. A. "Models, Mechanisms and Clinical Evidence for Cancer Dormancy." Nat Rev Cancer 7, no. 11 (2007): 834-46.

89. Aguirre Ghiso, J. A., K. Kovalski, and L. Ossowski. "Tumor Dormancy Induced by Downregulation of Urokinase Receptor in Human Carcinoma Involves Integrin and Mapk Signaling." J Cell Biol 147, no. 1 (1999): 89-104.

90. Enderling, Heiko, Nava Almog, and Lynn Hlatky. Systems Biology of Tumor Dormancy: Springer New York, 2013.

91. Marino, N., S. Woditschka, L. T. Reed, J. Nakayama, M. Mayer, M. Wetzel, and P. S. Steeg. "Breast Cancer Metastasis: Issues for the Personalization of Its Prevention and Treatment." Am J Pathol 183, no. 4 (2013): 1084-95.

92. Naoko, Takebe, Nguyen Dat, and Sherry X Yang. "Targeting Notch Signaling Pathway in Cancer: Clinical Development Advances and Challenges." Pharmacol Ther 141, no. 2 (2014): 140-49.

93. Locatelli, M. A., P. Aftimos, E. C. Dees, P. M. LoRusso, M. D. Pegram, A. Awada, B. Huang, R. Cesari, Y. Jiang, M. N. Shaik, K. A. Kern, and G. Curigliano. "Phase I Study of the Gamma Secretase Inhibitor Pf-03084014 in Combination with Docetaxel in Patients with Advanced Triple-Negative Breast Cancer." Oncotarget 8, no. 2 (2017): 2320-28.

94. Piha-Paul, S. A., P. N. Munster, A. Hollebecque, G. Argiles, O. Dajani, J. D. Cheng, R. Wang, A. Swift, A. Tosolini, and S. Gupta. "Results of a Phase 1 Trial Combining Ridaforolimus and Mk-0752 in Patients with Advanced Solid Tumours." Eur J Cancer 51, no. 14 (2015): 1865-73. 
95. Krop, I E, M Kosh, I Fearen, J Savoie, A Dallob, C Matthews, J Stone, E Winer, S J Freedman, and P Lorusso. "Phase I Pharmacokinetic (Pk), and Pharmacodynamic (Pd) Trial of the Novel Oral Notch Inhibitor Mk-0752 in Patients (Pts) with Advanced Breast Cancer (Bc) and Other Solid Tumors." Annals of Oncology 22, no. 6 (2006): 1413-9.

96. Locatelli, M., and G. Curigliano. "Notch Inhibitors and Their Role in the Treatment of Triple Negative Breast Cancer: Promises and Failures." Current Opinion in Oncology 29, no. 6 (2017): 411. 